\title{
Variación espacial y temporal en la composición de la dieta de peces invertívoros en un río neotropical, Venezuela
}

\author{
Mario Ortaz, Ricardo Martín \& Adriana López-Ordaz \\ Instituto de Biología Experimental, Universidad Central de Venezuela. Apartado postal 48170, Caracas 1041A, \\ Venezuela; maortaz@hotmail.com,ri14782@yahoo.es, adri_lopezordaz@yahoo.es
}

Recibido 10-VIII-2010. C Corregido 03-I-2011. Aceptado 26-I-2011.

\begin{abstract}
Spatial and temporal variation in diet composition of invertivore fishes in a tropical stream, Venezuela. Invertivores fishes are an important component of neotropical streams and they represent a link between aquatic invertebrates and piscivorous species. This study evaluated the breadth diet and interspecific food overlap of nine invertivores fish species during three consecutive hydrological phases: falling (December/07, January/08, February/08 and March/08), low (April/08) and rising waters (June/08), in two sections of a Venezuelan neotropical stream, which were located at different elevation, high watershed (HW) and low watershed (LW). The fishes were collected with a beach seine (5mm mesh) between 8:00 and 11:00 hours. The diet of each species was evaluated using an index of relative importance (IRI), which includes as variables the number, weight and occurrence frequency of food items consumed. The Levin' index $\left(\mathrm{B}_{\mathrm{i}}\right)$ and Morisita (IM) were used to estimate the breadth diet and interspecific food overlap, respectively. All estimations were made using the numeric proportion of preys. Nine fish species were captured, eight Characiformes, of which three were captured in HW (Knodus deuteronoides, Creagrutus bolivari and C. melasma) and five in LW (Thoracocharax stellatus, Moenkhausia lepidura, Cheirodon pulcher, Ctenobrycon spilurus and Aphyocharax alburnus), and one Cyprinodontiformes (Poecilia reticulata), which was also found in HW. In HW aquatic insects were the main resource consumed by fishes while plant material and terrestrial arthropods were secondary resources. In LW the fishes ingested all of these items in addition to zooplankton (Copepoda, Cladocera and larval stages of Decapoda). However, there was a temporal replacement with a predominance of zooplankton in falling and low water. In general, the breadth diet decreased during the falling water in both sections and increased in rising water. However, the average breadth diet was higher in HW. The interspecific food overlap was high in HW while low values were more frequent in LW and its temporal variation was opposed in both sections during almost all the sampling period. Rev. Biol. Trop. 59 (3): 1217-1231. Epub 2011 September 01.
\end{abstract}

Key words: trophic ecology, Characiformes, invertivores fishes, neotropical stream, Venezuela.

La ictiofauna invertívora es un componente conspicuo e importante de los ríos neotropicales ya que está conformado por un gran número de especies que ejercen una permanente presión depredadora sobre sus presas y representan una importante fuente de alimento para los piscívoros (Zaret \& Rand 1971, Winemiller 1990, Flecker 1992, Ortaz 1992, Taphorn 1992, Machado-Allison 2005, Winemiller 2005, Pouilly et al. 2006, TrujilloJiménez \& Toledo-Beto 2007).
En muchos ríos, los invertebrados presas están representados por una diversa comunidad de insectos que depende del material orgánico autóctono (perifiton y otras comunidades asociadas) y alóctono (Vannote et al. 1980, Wootton 1990, Allan 1995, Wetzel 2001, Barbee 2005). La disponibilidad de esta materia orgánica varía tanto a escala espacial como temporal, y está regulada por la disponibilidad de nutrientes, el tipo, desarrollo y fenología de la vegetación ribereña, las diferencias 
hidrogeomorfológicas del canal y las fluctuaciones en la descarga hidraúlica, entre otros (Vannote et al. 1980, Merritt \& Cummins 1984, Welcomme 1985, Lowe-McConnell 1987).

De manera general, se considera a la época de aguas bajas como la de menor disponibilidad de alimento para los peces invertívoros. Sin embargo, esto no necesariamente ocurre a todo lo largo de una cuenca ya que dependerá de las diferencias que existan en el gradiente altitudinal. Al respecto, en muchos ríos de tierras bajas se reduce en ésta época el transporte de materia orgánica desde río arriba y su importación local directa, lo cual produce una disminución en las abundancias poblacionales de los insectos bentónicos, además del efecto negativo directo de algunas variables ambientales (Thorp \& Delong 1994, Allan 1995). Sin embargo, en una sección de tierras altas con un bosque ribereño, los cambios pueden ser inclusive opuestos, de modo que las condiciones pueden favorecer el incremento en las abundancias de los insectos bentónicos (LoweMcConnell 1987, Allan 1995, Ortaz 2001). Es de esperar que esta variación altitudinal en la disponibilidad del bentos afecte las interacciones intra e interespecíficas de sus consumidores a través, por ejemplo, de la variación en la amplitud y la sobreposición de dieta de los peces invertívoros. Esta reducción en la amplitud y sobreposición de dieta en la zona del río donde disminuye la disponibilidad del bentos comenzaría al inicio de descenso de aguas y su menor valor debiera ocurrir cuando el río presenta su menor nivel hidrométrico. Adicionalmente, pueden ocurrir cambios importantes en la composición de la dieta ya que las condiciones ambientales podrían favorecer algunas comunidades de presas en particular.

Con base en lo anterior, en el presente trabajo se evaluó en un río neotropical en el que existe un evidente gradiente altitudinal (Ortaz 2001, Ortaz \& Rocha 2001, Martín 2007) si en la ictiofauna invertívora ocurre un cambio importante, a escala espacial y temporal, tanto en la composición de dieta como en su amplitud y sobreposición interespecífica.

\section{MATERIALES Y MÉTODOS}

Area de estudio: Se evaluaron dos secciones del cauce principal del río Orituco, ubicado en el centro-norte de Venezuela. Una de ellas correspondió a la subcuenca alta del río (SA, c.a $800 \mathrm{msnm}$ ) cerca del límite con el Parque Nacional Guatopo (1000'16.1" N-66²5'30.1" W) y la otra se ubicó en la subcuenca baja (SB, c.a $100 \mathrm{msnm}$ ), aproximadamente a $167 \mathrm{~km}$ aguas abajo y en línea recta de SA $\left(8^{\circ} 48^{\prime} 45.8^{\prime \prime}\right.$ $\left.\mathrm{N}-67^{\circ} 22^{\prime} 31^{\prime \prime} \mathrm{W}\right)$. En SA el río es uno típico de piedemonte de zona montañosa boscosa con un exuberante bosque de galería, el canal es permanente y su lecho está constituido por un sustrato inorgánico variado (Ortaz 2001). En $\mathrm{SB}$ el río drena en los Llanos altos venezolanos, la vegetación boscosa ribereña se reduce y predominan gramíneas y ciperáceas en algunas zonas, el canal es transitorio y su lecho está constituido principalmente por un sustrato areno-fangoso, con muy pocos cantos rodados y por la presencia de conglomerados ferruginosos (ripio) (Machado-Allison \& Moreno 1993).

Entre diciembre/07 y junio/08 se realizaron seis muestreos de campo, con una frecuencia mensual, que incluyeron las fases hidrológicas de descenso de aguas (diciembre/07, enero/08, febrero/08 y marzo/08), aguas bajas (abril/08) e inicio de ascenso de aguas (junio/08), las cuales se definieron de manera preliminar con base a la información meteorológica disponible. Para confirmar la fase hidrológica de cada sección, en cada muestreo se midió el ancho del canal (precisión: $0.01 \mathrm{~m}$ ), el perfil de profundidad (precisión: $0.01 \mathrm{~m}$ ) y la velocidad superficial de la corriente $(\mathrm{m} / \mathrm{s})$, con el método del flotador, con lo cual se calculó la descarga hidráulica $\left(\mathrm{m}^{3} / \mathrm{s}\right)$ (Wetzel \& Likens 2000).

Para la recolecta de los peces se empleó una red de cerco $(0.5 \mathrm{~cm}$ de abertura entre nudos). El esfuerzo de pesca en cada sección varió entre cuatro y diez lances, siendo seis el número más frecuente. La captura se realizó entre las 8:00 y 11:00 horas. En cada muestreo se realizó primero el trabajo en SA y al día siguiente en SB. Los peces recién capturados se preservaron en frío hasta su muerte, 
posteriormente en una solución de formol técnico al $10 \%$ y para su preservación definitiva en etanol técnico al $70 \%$.

Métodos: Los peces se identificaron según Géry (1977) y Taphorn (1992). Para cada ejemplar se estimó: la longitud estándar (precisión: $0.05 \mathrm{~mm}$ ) y el peso corporal húmedo (precisión: $0.01 \mathrm{~g}$ ). Posteriormente bajo lupa se extrajo el tracto digestivo. Se describió la composición de la dieta y se cuantificaron los distintos ítems para lo cual se emplearon las variables: presencia del ítem, número de individuos por ítem y peso de cada ítem (precisión: $0.001 \mathrm{~g}$ ), con las que se estimó un índice de importancia relativa (IIR) (Hyslop 1980, Ortaz et al. 2006). Los insectos acuáticos consumidos se identificaron según Merritt \& Cummins (1984), Roldán (1996) y Fernández \& Domínguez (2001).

Se estimó la amplitud de dieta con el índice de Levin $\left(\mathrm{B}_{\mathrm{i}}=1 / \Sigma \mathrm{P}_{\mathrm{ij}}{ }^{2}\right)$ (Chesson 1978, Pouilly et al. 2003, Pouilly et al. 2006). $\mathrm{P}_{\mathrm{ij}}=$ proporción numérica de la presa "j" en la dieta de la especie "i” y $\mathrm{N}=$ número de tipos de presas consumidas. La sobreposición trófica interespecífica se estimó con la modificación de Horn (1966) del índice de Morisita $\mathrm{IM}=2 \Sigma\left(\mathrm{P}_{\mathrm{ij}} \mathrm{P}_{\mathrm{kj}}\right)[\Sigma$ $\left.\left(\mathrm{P}_{\mathrm{ij}}{ }^{2}+\mathrm{P}_{\mathrm{kj}}{ }^{2}\right)\right]^{-1}$ (Zaret \& Smith 1984, Smith 1985). $\mathrm{P}_{\mathrm{ij}}=$ proporción numérica del ítem " $\mathrm{j}$ ” en la dieta de la especie "i" y $\mathrm{P}_{\mathrm{kj}}=$ proporción numérica del ítem "j" en la dieta de la especie "k".

\section{RESULTADOS}

Composición de la dieta: Las especies analizadas fueron: Knodus deuteronoides (SA) $(\mathrm{n}=224)$, Creagrutus bolivari (SA) $(\mathrm{n}=116)$, Creagrutus melasma (SA) ( $\mathrm{n}=60)$, Poecilia reticulata $(\mathrm{SA})(\mathrm{n}=49)$, Thoracocharax stellatus (SB) ( $\mathrm{n}=112)$, Moenkhausia lepidura (SB) $(\mathrm{n}=90)$, Cheirodon pulcher $(\mathrm{SB})(\mathrm{n}=38)$, Ctenobrycon spilurus (SB) $(\mathrm{n}=38)$ y Aphyocharax alburnus (SB) $(\mathrm{n}=28)$.

La dieta de las especies en SA estuvo constituida por invertebrados acuáticos (insectos, gastrópodos y ostrácodos), artrópodos terrestres y material vegetal alóctono. Los insectos representaron el grupo más importante ya que los otros aparecieron ocasionalmente en la dieta de sólo una especie ( $K$. deuteronoides). Los órdenes Coleoptera, Diptera, Ephemeroptera y Trichoptera fueron los más consumidos por los carácidos evaluados y sólo Diptera y Ephemeroptera fueron consumidos en una proporción importante por P. reticulata (Cuadro 1). Los artrópodos terrestres fueron un componente importante en $C$. bolivari y $K$. deuteronoides, mientras que en $C$. melasma y $P$. reticulata su consumo fue insignificante. El consumo de material vegetal alóctono fue alto en las cuatro especies y a lo largo del lapso evaluado. K. deuteronoides consumió el mayor número de items (13), seguida por C . bolivari (11), C. melasma (8) y $P$. reticulata (5). El consumo promedio de insectos acuáticos fue parecido entre los carácidos y bajo en $P$. reticulata. El consumo promedio de invertebrados terrestres fue muy bajo en $C$. melasma, representó cerca del $15 \%$ del consumo total en $C$. bolivari y $K$. deuterodonoides y no fue una presa depredada por $P$. reticulata. El consumo promedio de material vegetal alóctono fue alto en $P$. reticulata y menor en los carácidos.

La dieta de los invertívoros en SB consistió de invertebrados bentónicos (principalmente insectos), zooplancton (copépodos, cladóceros y estadios preadultos de crustáceos Decapoda), artrópodos terrestres y material vegetal alóctono (Cuadro 1). En general, aunque en SB la dieta estuvo constituida por un número parecido de items al encontrado en SA (12 en SB vs 13 en SA), hubo un cambio importante en su composición ya que sólo el $47 \%$ de las presas consumidas fueron comunes para ambas secciones. En SB no se consumieron presas como Ephemeroptera, Gastropoda, Megaloptera, Odonata y Plecoptera, algunas de las cuales fueron importantes en SA. Adicionalmente, en SB se consumieron otros grupos como Cladocera, Copepoda, Collembola y Decapoda. El consumo promedio de insectos acuáticos en SB fue menor al encontrado en SA y representó aproximadamente el 19\% del total de items consumidos. Entre los insectos, Diptera fue el más consumido tanto por los valores de consumo como por el número de especies que 
CUADRO 1

Porcentaje IIR de los ítems alimenticios por especie

TABLE 1

Percentage IRI of food item for each species

$\begin{array}{cccccccccc}\text { Item } & \text { Cbo } & \text { Cme } & \text { Kde } & \text { Pre } & \text { Cts } & \text { Mol } & \text { Apa } & \text { Chp } & \text { Ths } \\ \text { At } & 14.9 & 0.6 & 13.2 & 0 & 0 & 14.1 & 3.4 & 0 & 86.5 \\ \text { Ca } & 0 & 0 & 0 & 0 & 25.6 & 21.3 & 28.3 & 41.6 & 0 \\ \text { Cl } & 0 & 0 & 0 & 0 & 0 & 1.5 & 3.4 & 0 & 0 \\ \text { Co } & 11.1 & 0.7 & 4.1 & 0 & 5.4 & 4.4 & 0 & 0 & 5.4 \\ \text { Cp } & 0 & 0 & 0 & 0 & 4.1 & 1.0 & 15.9 & 0 & 0 \\ \text { De } & 0 & 0 & 0 & 0 & 0.3 & 1.0 & 8.4 & 0 & 0 \\ \text { Di } & 15.3 & 17.7 & 22.8 & 14.2 & 6,0 & 8.4 & 14.5 & 1.9 & 2.0 \\ \text { Ef } & 14.9 & 21.5 & 15.7 & 14.1 & 0 & 0 & 0 & 0 & 0 \\ \text { Ga } & 0 & 0 & 0.5 & 0 & 0 & 0 & 0 & 0 & 0 \\ \text { He } & 0.8 & 1.5 & 1.6 & 0 & 0 & 2.4 & 0 & 0 & 1.1 \\ \text { Is } & 0.3 & 0 & 0.3 & 0 & 0 & 0.4 & 0 & 0 & 0 \\ \text { Ma } & 29 & 38.2 & 31.3 & 77.5 & 50.9 & 42.6 & 21.2 & 50.5 & 2.4 \\ \text { Me } & 0.3 & 0 & 1.1 & 0 & 0 & 0 & 0 & 0 & 0 \\ \text { Od } & 0.6 & 0 & 0.8 & 0.5 & 0 & 0 & 0 & 0 & 0 \\ \text { Os } & 0 & 0 & 0.3 & 0 & 0.3 & 2.8 & 0 & 4.1 & 2.6 \\ \text { Pl } & 0.3 & 1.5 & 1.4 & 0 & 0 & 0 & 0 & 0 & 0 \\ \text { Tr } & 15.4 & 18.3 & 7.2 & 0.8 & 7.3 & 0 & 0 & 1.9 & 0\end{array}$

Cbo: C. bolivari. Cme: C. melasma. Kde: K. deuteronoides. Pre: P. reticulata. Cts: C. spilurus. Mol: M. lepidura. Apa: A. alburnus. Chp: C. pulcher. Ths: T. stellatus. At: artrópodo terrestre. Ca: cladócero. Cl: colémbolo. Co: coleóptero. Cp: copépodo. De: larva decápodo. Di: díptero. Ef: efemeróptero. Ga: gastrópodo. He: hemíptero. Is: isópodo. Ma: material alóctono vegetal. Me: megalóptero. Od: odonato. Os: ostrácodo. Pl: plecóptero. Tr: tricóptero.

lo depredaron. Otro componente bentónico que apareció con cierta frecuencia fueron los ostrácodos, cuyo consumo en SA fue muy bajo y lo ejerció $K$. deuteronoides y sólo en un mes. En SB la depredación sobre el zooplancton fue importante en buena parte del registro, el cual nunca formó parte de la dieta en SA. El consumo promedio de zooplancton nunca fue inferior al $25 \%$ en cuatro de las cinco especies evaluadas y este fue parecido en A. alburnus y C. pulcher. Sólo en T. stellatus no se encontró evidencia de depredación sobre el zooplancton. Los cladóceros fue el grupo del zooplancton más depredado en las cuatro especies y sólo en A. alburnus los copépodos llegaron a representar un porcentaje alto. El consumo de preadultos de Decapoda fue bajo en las especies evaluadas. Los artrópodos terrestres representaron en SB un recurso relativamente poco importante en la mayoría de las especies con excepción de T. stellatus para la cual representó su principal fuente de alimento. Al igual que en SA, en SB el material vegetal alóctono fue un recurso importante y su promedio global no fue inferior al $25 \%$, con excepción de lo obtenido en T. stellatus. De hecho en especies como $C$. pulcher llegó a representar hasta la totalidad de su dieta (junio/08). M. lepidura fue la especie que consumió el mayor número de tipos de presas y en casi la totalidad del registro consumió, de manera simultánea, presas del bentos, plancton, artrópodos terrestres y material vegetal alóctono. Por el contrario, las dietas de $C$. pulcher y $T$. stellatus fueron las más estrechas mientras que las de A. alburnus y $C$. spilurus mostraron una condición intermedia. 
En SA se observó un consumo alto de insectos acuáticos a lo largo de las fases evaluadas y el consumo de invertebrados terrestres se mantuvo bajo y poco variable (Fig. 1). Por el contrario, en SB hubo una progresiva disminución en el consumo de insectos acuáticos desde el inicio de descenso de aguas hasta aguas bajas con un posterior incremento en ascenso de aguas. El zooplancton representó en SB la principal comunidad depredada en buena parte del registro y su consumo disminuyó progresivamente hasta desaparecer al inicio de ascenso de aguas. El consumo de invertebrados terrestres mostró un ligero incremento en el lapso evaluado y resultó mayor al obtenido en SA, fundamentalmente debido a T. stellatus.

Amplitud de dieta: En C. bolivari, la menor amplitud se obtuvo en aguas bajas (abril/08) y la mayor en descenso de aguas (enero/08). La menor amplitud no correspondió con una reducción general del consumo de presas y, por el contrario, el promedio de presas consumidas en este mes fue elevado (X: 7.1 presas/pez), y solo superado por el obtenido en marzo (X: 8.2 presas/pez). En este caso sólo un tipo de presa (Trichoptera) representó el $72 \%$ de todas las consumidas, que en este mes
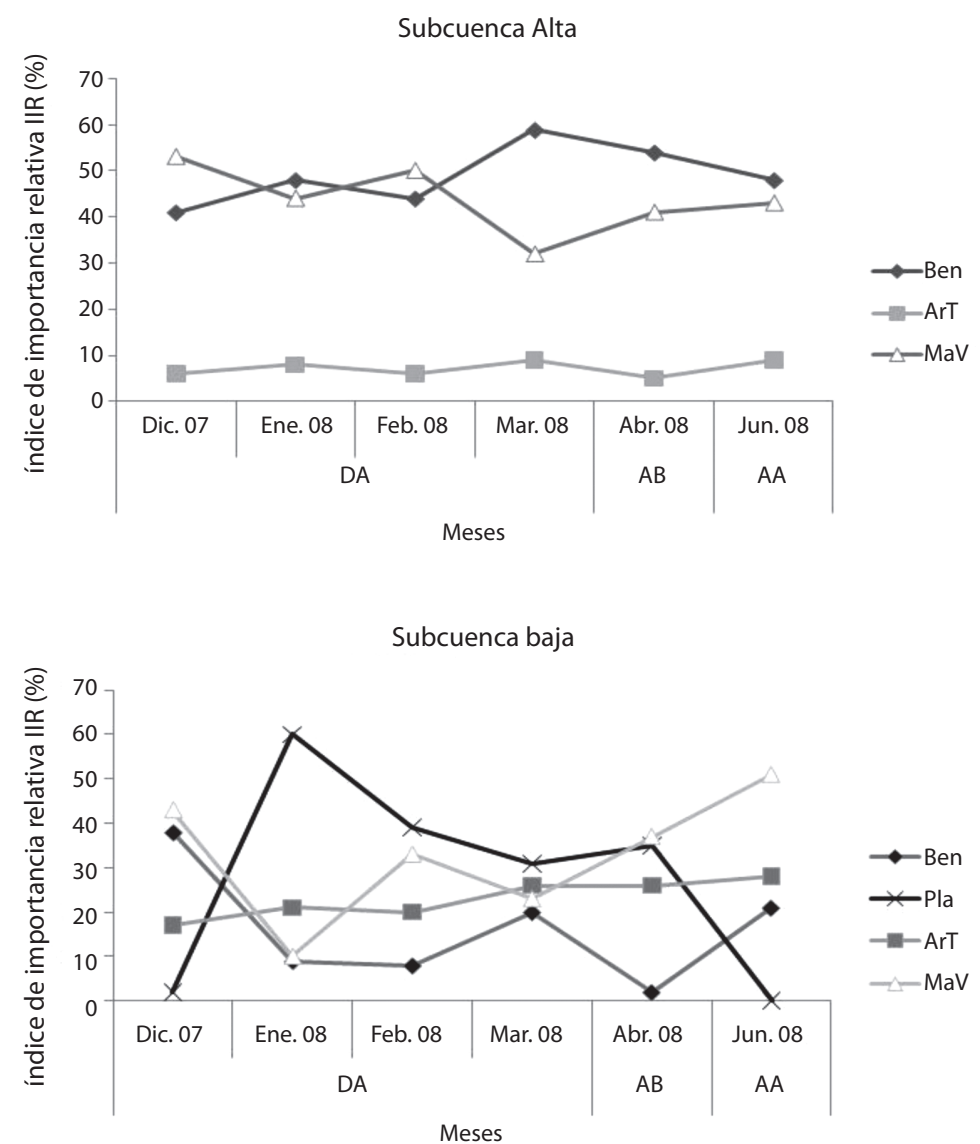

Fig. 1. Porcentaje IIR de los ítems consumidos por la ictiofauna invertívora. ArT: artrópodos terrestres. Ben: Bentos. Pla: Plancton. MaV: Material vegetal alóctono. DA: descenso de aguas. AB: aguas bajas. AA: ascenso de aguas.

Fig. 1. Percentage IRI of food items ingested by invertivores fishes. 
incluyó a Ephemeroptera (9.8\%), Artrópodos terrestres $(5.5 \%)$, Coleoptera (4.5\%), Diptera (3.2\%), Odonata $(2.5 \%)$ y Hemiptera $(2.5 \%)$. El mayor valor correspondió con un bajo consumo de presas (X: 3.8 presas/pez) pero de manera más proporcional y varió entre $2.2 \%$ (Megaloptera y Plecoptera) y 26.7\% (Diptera y Artrópodos terrestres). En C. melasma, el menor valor se obtuvo en marzo/08 (descenso de aguas) y el mayor en junio/08 (ascenso de aguas) (Cuadro 2). La tendencia general obtenida consistió en un descenso progresivo de diciembre/07 hasta marzo/08, con excepción de febrero/08 y a partir de marzo/08 incrementó hasta ascenso de aguas (junio/08). La menor amplitud obtenida correspondió con un elevado promedio de presas consumidas (X: 20.2 presas/pez) pero de manera poco proporcional ya que sólo Diptera representó casi el $90 \%$ del total de presas consumidas (89.1\%) mientras que el $10 \%$ restante quedó repartido entre Trichoptera (6.9\%), Ephemeroptera $(2.9 \%)$ y Artrópodos terrestres (1.1\%). La mayor amplitud obtenida correspondió con un bajo promedio de presas consumidas (X: 1.3 presas/pez) de manera más proporcional y varió entre $25 \%$, para Ephemeroptera y $37.5 \%$ para Diptera y Trichoptera. En K. deuteronoides la tendencia general fue una disminución progresiva a lo largo de descenso de aguas, con excepción de un leve incremento en enero/08, con un incremento importante en aguas bajas y una nueva disminución en ascenso de aguas. En esta especie la menor amplitud de dieta se obtuvo en marzo/08 (descenso de aguas) y la mayor en abril/08 (aguas bajas). En el mes de menor amplitud de dieta el consumo de presas fue elevado (X: 7.1 presas/pez) y el consumo de las presas fue poco proporcional ya que sólo Diptera representó el $67 \%$ del total y el resto quedó repartido entre Ephemeroptera (12\%), Artrópodos terrestres (9\%), Trichoptera (6\%) y un último grupo constituido por Coleoptera, Hemiptera y Odonata (6\% restante). La mayor amplitud correspondió, igualmente, con un elevado consumo de presas (X: 5.1 presas/pez) pero más uniforme ya que la variación de la proporción de las cuatro presas más depredadas fue de sólo $9 \%$ (Trichoptera: 16\%, Diptera: 21\%, Artrópodos terrestres: 22\% y Ephemeroptera: $25 \%$ ) mientras que el resto representaron en conjunto el $16 \%$ del total. $P$. reticulata fue el invertívoro con el consumo más bajo de invertebrados acuáticos y terrestres $\mathrm{y}$, entre diciembre/07 y febrero/08, su dieta consistió exclusivamente de material vegetal alóctono y sólo entre marzo/08 y junio/08 depredó insectos acuáticos pero sin excluir al material

CUADRO 2

Amplitud de dieta por especie $\left(\mathrm{B}_{\mathrm{i}}\right)$ en el periodo muestral (abreviatura en Cuadro 1)

TABLE 2

Breadth diet values for each species $\left(\mathrm{B}_{\mathrm{i}}\right)$ in sampling period (abbreviation in Table 1)

\begin{tabular}{|c|c|c|c|c|c|c|}
\hline & Dic/07 & Ene/08 & $\mathrm{Feb} / 08$ & Mar/08 & $\mathrm{Abr} / 08$ & Jun/08 \\
\hline Cbo & 4.48 & 5.76 & 4.45 & 3.59 & 1.87 & 4.68 \\
\hline Cme & 2.66 & 1.71 & 2.24 & 1.25 & 2.05 & 2.91 \\
\hline Kde & 3.66 & 4.05 & 3.62 & 2.05 & 5.10 & 3.31 \\
\hline Pre & ne & ne & ne & 1.0 & 2.0 & 2.32 \\
\hline Cts & 1.47 & 1.01 & 1.03 & ne & ne & $\mathrm{Ne}$ \\
\hline Mol & 1.81 & 1.38 & 1.19 & 1.63 & 1.22 & 3.55 \\
\hline Apa & ne & 1.11 & 1.46 & 1.01 & ne & 3.0 \\
\hline Chp & ne & 1.01 & 1.25 & ne & 1.0 & $\mathrm{Ne}$ \\
\hline Ths & 3.13 & 1.0 & 1.14 & 1.17 & 1.0 & 1.0 \\
\hline
\end{tabular}

ne: no estimado. 
vegetal. La menor amplitud de dieta se obtuvo en marzo/08, en el que fue alto el consumo de insectos acuáticos (X: 4.5 presas/pez). Este bajo valor coincidió con el consumo de un solo tipo de presa (Diptera). La mayor amplitud de dieta se obtuvo en junio/08 y coincidió con un consumo relativamente alto $\left(\mathrm{X}_{\text {junio }}: 3\right.$ presas/ pez) y un incremento en la variedad de presas que incluyó Diptera (50\%), Ephemeroptera $(41 \%)$ y Trichoptera (9\%).

La amplitud de dieta obtenida en C. boliva$r i$ fue superior a la del resto de las especies de SA, con excepción de lo obtenido en abril/08.

En $C$. spilurus, la menor amplitud de dieta se obtuvo en enero/08 y la mayor en diciembre/07 (ambas en descenso de aguas). Esta especie no se capturó entre marzo/08 y junio/08. La baja amplitud en enero/08 correspondió con un elevado consumo de Cladocera, quien representó el $99.5 \%$ de su dieta, mientras que el resto de las presas representaron el 0.5 $\%$ (Decapoda: $0.1 \%$, Copepoda: $0.2 \%$, Diptera: $0.1 \%$ y Ostracoda: $0.1 \%$ ). La mayor amplitud correspondió con el consumo de sólo 2 presas pero en proporciones distintas (Trichoptera: 80\% y Cladocera: 20\%). En A. alburnus la menor amplitud se obtuvo en marzo/08 (aguas bajas) y la mayor en junio/08 (ascenso de aguas). Esta especie no se capturó en diciembre/07 y abril/08. La reducción de la amplitud en marzo/08 correspondió con el consumo de sólo 2 tipos de presas y en proporciones muy distintas (Copepoda: $99.4 \%$ del total y Cladocera: $0.6 \%$ ), mientras que la mayor amplitud no correspondió con un incremento importante en el número de tipos de presas consumidas (Diptera, Collembola y Artrópodos terrestres) pero si en la proporción de consumo (33.3\% para cada una). En M. lepidura, la mínima amplitud se obtuvo en febrero/08 (descenso de aguas) y la máxima en junio/08 (ascenso de aguas). La baja amplitud no correspondió con una disminución del número de tipos de presas consumidas sino con la variación en sus proporciones, que en este caso fue elevada para Cladocera $(91.66 \%)$ y muy baja para el resto (Ostracoda: 4.16\%, Diptera: $2.77 \%$ y Artrópodos terrestres: $1.41 \%)$. La mayor amplitud correspondió con un consumo más proporcional de las presas (Artrópodos terrestres: $37.5 \%$, Diptera: 25\%, Coleoptera: $25 \%$ y Collembola: $12.5 \%$ ). En este mes, el número de tipos de presas consumidas fue similar al encontrado en el mes con la dieta más estrecha. La menor amplitud en $C$. pulcher se obtuvo en aguas bajas y se debió al consumo de un solo tipo de presa (Cladocera). Esta especie no se capturó en todo el registro evaluado. La mayor amplitud se obtuvo en descenso de aguas y fue consecuencia del consumo de una mayor variedad de presas con intensidades de depredación parecidas (Cladocera: $44.9 \%$, Diptera: $7.7 \%$, Ostracoda: $7.7 \%$ y Trichoptera: $7.7 \%$ ). La dieta de T. stellatus fue la más especializada, como en $C$. pulcher, pero en un mayor número de meses (enero/08, abril/08 y junio/08), su amplitud de dieta fue mínima debido al consumo de un solo tipo de presa. Su mayor amplitud se obtuvo al inicio de descenso de aguas y fue consecuencia del consumo de una mayor variedad de presas con intensidades de consumo variables (Hemiptera: 6.8\%, Diptera: $12.2 \%$, Coleoptera: $32.1 \%$ y Artrópodos terrestres: $34.3 \%$ ).

En la mayor parte del registro, la amplitud de dieta de M. lepidura fue superior, con excepción de $T$. stellatus en diciembre/07 y A. alburnus en febrero/08. En general, la variación en A. alburnus y $M$. lepidura fue parecida y consistió en una disminución progresiva desde descenso hasta aguas bajas, con excepción de un comportamiento opuesto en febrero/08, para luego incrementar en ascenso de aguas.

La variación general de la amplitud de dieta fue parecida en varias especies y consistió en una disminución a medida que progresaron las fases de descenso y aguas bajas, con un mínimo que varió dependiendo de la especie y no de la sección. En C. bolivari en SA este mínimo ocurrió en aguas bajas, mientras que en el resto de las especies de esta sección y en M. lepidura en SB este ocurrió en descenso de aguas. En el resto de las especies de SB la menor amplitud se obtuvo tanto en descenso como en aguas bajas. Otro resultado parecido en ambas secciones fue el incremento importante en la amplitud de dieta en ascenso de 
aguas lo cual ocurrió en todas las especies, con excepción de T. stellatus. La amplitud promedio de dieta resultó mayor en SA (Fig. 2).

Sobreposición interespecífica de dieta: Los valores de sobreposición de dieta en SA resultaron altos $(>50 \%)$ en buena parte del registro (Cuadro 3). En esta sección, la sobreposición tendió a ser alta entre los carácidos $(C$. bolivari, C. melasma y K. deuteronoides) y baja entre éstos y $P$. reticulata, de hecho el menor valor se obtuvo entre $C$. bolivari y $P$. reticulata en aguas bajas (abril/08). La sobreposición no fue recurrentemente alta en un único par de especies y por el contrario se obtuvieron valores altos en todos los pares comparados. Los bajos valores se obtuvieron en enero/08 y abril/08 (IM media: $57.1 \%$ y IM media: $52 \%$, respectivamente), y el mayor se obtuvo al inicio del descenso de aguas (diciembre/07) (IM media $: 91.6 \%$ ) (Fig. 3). La variación en los pares $C$. bolivari - C. melasma y $C$. bolivari $-K$. deuteronoides fue parecida entre diciembre/07 y febrero/08 y opuesta en el resto del período. En el par C. melasma - K. deuteronoides y en el período diciembre/07 - marzo/08 la variación fue más parecida a la que ocurrió entre C. bolivari - C. melasma en el mismo lapso, mientras que en abril/08 y junio/08 varió tal y como ocurrió en $C$. bolivari - K. deuteronoides.

Subcuenca Alta

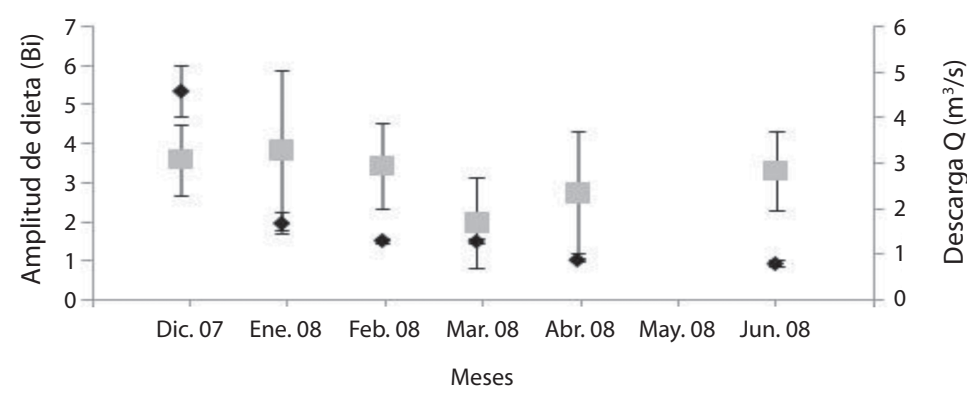

$-\mathrm{Bi} \bullet \mathrm{Q}$

Subcuenca baja

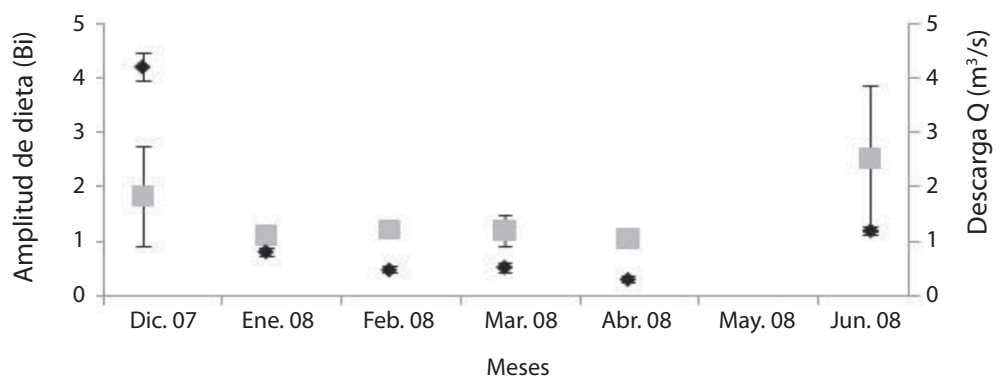

$B \mathrm{Bi} \bullet \mathrm{Q}$

Fig. 2. Amplitud de dieta (valor medio de $\mathrm{B}_{\mathrm{i}}$ ).

Fig. 2. Breadth diet (median values of $B_{i}$ ). 
CUADRO 3

Sobreposición de dieta entre pares de especies (abreviatura en el Cuadro 1)

TABLE 3

Interspecific food overlap between pair of species (abbreviation in Table 1)

\begin{tabular}{|c|c|c|c|c|c|c|}
\hline & Dic/07 & Ene/08 & $\mathrm{Feb} / 08$ & Mar/08 & $\mathrm{Abr} / 08$ & Jun/08 \\
\hline (Cbo-Cme) & 90.9 & 35.4 & 59.2 & 78.1 & 94.8 & 74.8 \\
\hline (Cbo-Kde) & 90.5 & 87.2 & 94.6 & 87.4 & 44.8 & 91.2 \\
\hline (Cbo-Pre) & ne & ne & ne & 68.7 & 12.6 & 68.5 \\
\hline (Cme-Kde) & 93.3 & 48.7 & 47.6 & 95.1 & 56.7 & 62 \\
\hline (Cme-Pre) & ne & ne & ne & 99 & 35.3 & 80.7 \\
\hline (Kde-Pre) & ne & ne & ne & 91.1 & 67.5 & 72.9 \\
\hline (Apa-Cts) & 0 & 99.7 & 13.4 & 0 & ne & $\mathrm{Ne}$ \\
\hline (Apa-Mol) & 18.4 & 98.8 & 8.9 & 0.6 & 99.3 & 81.4 \\
\hline (Apa-Chp) & ne & 99.7 & 8.9 & ne & 100 & $\mathrm{Ne}$ \\
\hline (Apa-Ths) & 25.3 & 0 & 0 & 0 & 0 & 49.9 \\
\hline (Cts-Mol) & 0 & 98.3 & 1.4 & 2.5 & ne & $\mathrm{Ne}$ \\
\hline (Cts-Chp) & ne & 99.9 & 1.4 & ne & ne & $\mathrm{Ne}$ \\
\hline (Cts-Ths) & 0 & 0 & 0 & 0 & ne & $\mathrm{Ne}$ \\
\hline (Mol-Chp) & ne & 98.3 & 99.8 & ne & 99.3 & $\mathrm{Ne}$ \\
\hline (Mol-Ths) & 76.6 & 0.9 & 1.9 & 12.2 & 0 & 58.5 \\
\hline (Chp-Ths) & ne & 0 & 0.3 & ne & 0 & $\mathrm{Ne}$ \\
\hline
\end{tabular}

ne: no estimado.

En C. bolivari - C. melasma la sobreposición fue alta al inicio de descenso (diciembre/07) y en aguas bajas (abril/08) y sólo en enero/08 fue menor del 50\%. Entre C. bolivari - K. deuteronoides fueron frecuentes los valores altos ( $>80 \%$ en todos los meses evaluados), con excepción de aguas bajas (abril/08) $(<50 \%)$. $\mathrm{Al}$ igual que entre $C$. bolivari y C. melasma, la sobreposición fue alta al inicio de descenso de aguas (diciembre/07) pero también en descenso (febrero/08) y ascenso de aguas (junio/08). La sobreposición entre $C$. melasma y $K$. deuteronoides tendió a ser menor ya que fueron más frecuentes los valores menores al $60 \%$, sin embargo, también fue alta al inicio de descenso (diciembre/07) y en aguas bajas (marzo/08). En $P$. reticulata y debido a que no consumió invertebrados entre diciembre/07 y febrero/08, sólo se pudo estimar la sobreposición de dieta con los carácidos capturados en el resto del período. Su dieta tendió a ser diferente a la de $C$. bolivari por su baja sobreposición y fue parecida a la de $C$. melasma y $K$. deuteronoides en marzo/08 y junio/08 y baja en abril/08. No se observó una relación entre la sobreposición y el porcentaje de presas comunes ya que, por ejemplo, en meses en los que éste fue bajo, como entre $C$. bolivari y $C$. melasma en diciembre/07, enero/08, febrero/08 y abril/08, se obtuvieron por igual valores bajos y altos de sobreposición. Lo mismo ocurrió en otros pares como $C$. melasma y $K$. deuteronoides, en los que el porcentaje de presas comunes fue parecido en parte del lapso evaluado (ca 40\%), y sin embargo la variación de la sobreposición de dieta fue grande. Aunque no se obtuvo relación entre estas variables, el porcentaje de presas comunes siempre fue alto entre $C$. bolivari y $K$. deuteronoides y bajo entre $P$. reticulata y los carácidos. Tampoco se observó una relación entre la sobreposición de dieta y el promedio de presas consumidas/especie, ya que, por ejemplo, el mayor promedio obtenido en marzo/08, no correspondió con una alta sobreposición 


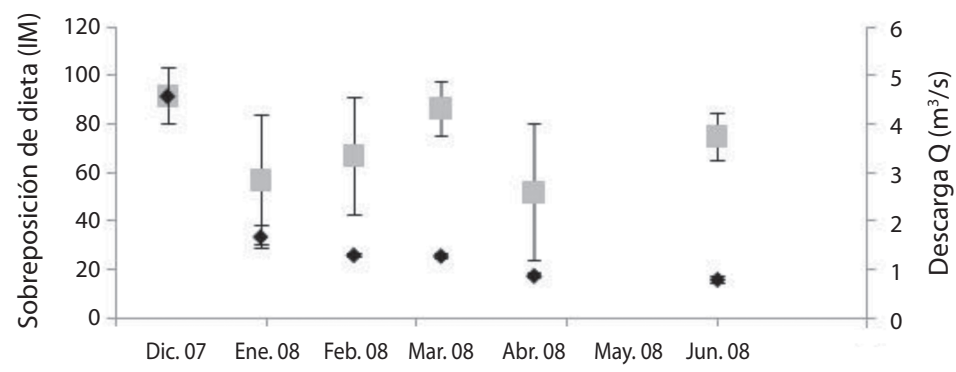

Meses

$\mathrm{IM} \bullet \mathrm{Q}$

Subcuenca baja

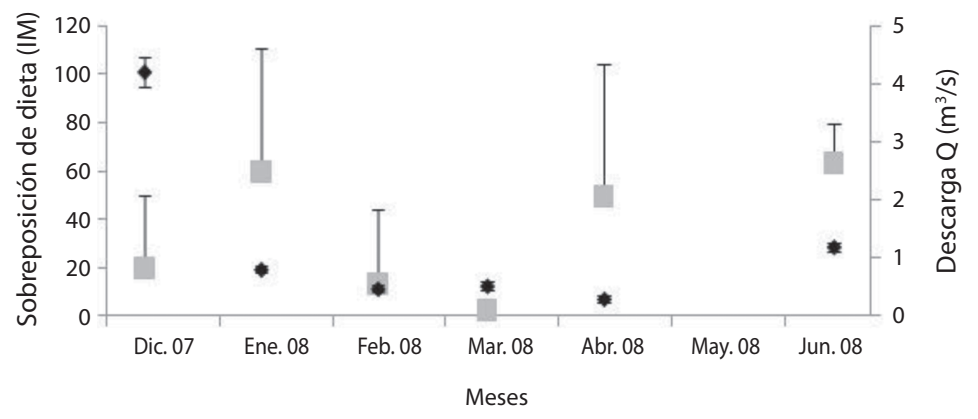

$\mathbb{I} \mathrm{IM} \bullet \mathrm{Q}$

Fig. 3. Sobreposición trófica interespecífica (valor medio de IM).

Fig. 3. Interspecific food overlap (median values of IM).

de dietas. Igualmente, los bajos promedios de presas consumidas no correspondieron necesariamente con bajos valores de sobreposición.

En SB se obtuvieron, al igual que en SA, valores de sobreposición superiores al 50\%, sin embargo lo frecuente fueron valores bajos. En enero/08 (descenso de aguas) se obtuvieron valores altos en la mayoría de los pares comparados así como entre A. alburnus y $M$. lepidura en abril/08 (aguas bajas) y junio/08 (ascenso de aguas), el cual fue consecuencia del consumo simultáneo y elevado de cladóceros. Lo mismo ocurrió en abril/08 entre A. alburnus y
M. lepidura. La variación de la sobreposición de dieta entre diciembre/07 y marzo/08 fue parecida en los pares A. alburnus - C. spilurus, A. alburnus - M. lepidura y C. spilurus - M. lepidura (Cuadro 3) y fue baja al inicio de descenso de aguas (diciembre/07), incrementó en enero/08 y disminuyó hasta marzo/08. La sobreposición entre A. alburnus y M. lepidura fue alta en descenso, aguas bajas y ascenso de aguas. En el par M. lepidura - C. pulcher, todos los valores fueron altos, sin embargo en este caso el número de individuos analizados de $C$. pulcher en abril/08 fue bajo y probablemente 
no representó el comportamiento de la población. La dieta de $T$. stellatus fue la más disímil en SB lo cual produjo muy bajos valores de IM e inclusive en muchas comparaciones se obtuvo el mínimo valor posible. Sólo con $M$. lepidura, esta especie sobrepuso su dieta en más del $50 \%$ pero sólo al inicio de descenso y ascenso de aguas.

En SA ca del $80 \%$ de los estimados de IM fueron superiores al $50 \%$ mientras que en SB esto se obtuvo sólo en el $28 \%$ de los casos (Fig. 3). En SB tampoco se obtuvo una relación entre la sobreposición de dieta y el porcentaje de presas comunes y el promedio de presas consumidas. En casi todo el período evaluado se encontró una tendencia opuesta en la variación temporal de la sobreposición trófica interespecífica promedio entre secciones.

\section{DISCUSIÓN}

En descenso y aguas bajas, las presas más consumidas en $\mathrm{SB}$, con excepción de $T$. stellatus, no fueron los insectos acuáticos sino el zooplancton. Esto podría ser consecuencia de la disminución en la disponibilidad de insectos acuáticos bentónicos por condiciones ambientales adversas como la reducción del nivel hidrométrico del canal y la difusión de oxígeno por la disminución de la turbulencia, entre otros. Por el contrario, el consumo de zooplancton en estos meses, podría deberse al incremento en su densidad (abundancia/ volumen) por la disminución del volumen de agua transportada, sumado al aumento en la abundancia del alimento, ya que en estos meses se observaron mantos perifíticos en las riberas del canal los cuales pueden ser una fuente adicional de alimento junto al fitoplancton en tránsito (Calow \& Petts 1992, Allan 1995, Wetzel 2001). Otro factor favorable para el zooplancton en estas fases fue la disminución considerable del caudal, de modo que las tasas de recambio poblacional podrían superar las pérdidas de individuos por efecto del arrastre. Este resultado coincide parcialmente con el obtenido por Martín (2007) para la misma sección quien reportó bentos, plancton, necton y material de origen terrestre en la dieta de $A$. alburnus, $C$. spilurus y M. lepidura en ascenso $\mathrm{y}$ aguas bajas mientras que en descenso no hubo un consumo de zooplancton. En SA, condiciones como la mayor variedad de sustratos inorgánicos, producen un lecho físicamente más heterogéneo, una menor reducción temporal del caudal, una permanente turbulencia que garantiza la adecuada oxigenación de los distintos estratos y el permanente ingreso de materia orgánica. Éstas condiciones son adecuadas para la comunidad de insectos acuáticos bentónicos, los cuales representan en esta sección la principal fuente de alimento de los invertívoros. Además, en descenso y aguas bajas se reduce el arrastre de individuos por la menor descarga (Moyle \& Senanayake 1984, Allan 1995, Ortaz 2001).

Los cambios observados indican que dentro de un mismo gremio trófico y en el mismo ambiente puede ocurrir una variación importante en el tipo de presa consumida y en su intensidad de depredación. Estos cambios ocurren tanto a una escala espacial (altitudinal) como temporal (entre fases hidrológicas) y en buena medida son inducidos inicialmente por los cambios en la descarga hidráulica, los cuales pueden resultar parcialmente amortiguados por la importación de material orgánico terrestre que representa un pulso importante de alimento para algunas presas de los peces invertívoros como los insectos acuáticos y/o una fuente directa de alimento para los propias invertívoros como fue el caso de T. stellatus quien consumió fundamentalmente insectos terrestres. Una especie abundante en SB fue $M$. lepidura la cual si bien consumió principalmente presas autóctonas, depredó, como T. stellatus, a los invertebrados terrestres y de hecho, fue la que sobrepuso más su dieta con ésta (IM media $_{18 \%}$ ) y compartió el mayor porcentaje de presas comunes (X:29\%).

$\mathrm{Si}$ bien los invertebrados son un recurso de uso frecuente, su importancia varía dependiendo del ambiente. Pouilly et al. (2006) encontraron en la cuenca amazónica del río Beni (Bolivia) que aunque los invertívoros fue el grupo que dominó o co-dominó junto con los 
detritívoros, su número de especies se redujo con la disminución de la altitud e incrementó el número de detritívoros, algívoros y piscívoros. Por otra parte, los autores no encontraron un consumo importante de zooplancton, contrario a lo reportado para otros ambientes (Mérigoux \& Porton 1998), incluyendo el presente estudio.

En SB, M. lepidura fue la que mostró la dieta más amplia debido a la mayor variedad de tipos de presas ingeridas y a la mayor intensidad y proporcionalidad en su consumo. Este resultado fue equivalente al obtenido en SA para $K$. deuteronoides y $C$. bolivari. $C$. pulcher fue un caso interesante y un ejemplo de una especie con una gran plasticidad de dieta que consume invertebrados acuáticos, material vegetal y perifiton (Angermeier \& Karr 1983, Pouilly et al. 2006).

En ambas secciones la amplitud promedio de dieta disminuyó a medida que progresaron las fases de descenso y aguas bajas, con un incremento importante en ascenso de aguas, que en SB consistió en un reemplazo del zooplancton por los insectos acuáticos. Además, los valores fueron mayores en SA. La variación en el número y en la proporción de consumo de las presas no tuvo el mismo efecto sobre el estimado de amplitud de dieta. Por ejemplo, la amplitud en $C$. bolivari en abril/08 disminuyó considerablemente, y no se debió a una reducción del consumo de presas, ya que inclusive éste fue proporcionalmente mayor al de otros meses en los que su dieta fue más amplia. $\mathrm{La}$ causa de esta reducción fue el consumo menos equitativo de las presas puesto que seis de ellas representaron el 22\% de su dieta y sólo una (Trichoptera) el 72\%. Algo parecido ocurrió con $K$. deuteronoides en marzo/08, mes en el que, aunque el promedio de presas consumidas fue el más alto del registro (X: 7 presas/pez), éste fue desproporcionado. El elevado consumo de sólo un tipo de presa también explicó la reducción de la dieta de C. melasma y A. alburnus en marzo/08 (Diptera: 89.1\%, Copepoda: 99.4\%, respectivamente), de C. spilurus en enero/08 (Cladocera: $99.5 \%$ ) y de M. lepidura en febrero/08 (Cladocera: 91.7\%). La reducción extrema en $C$. pulcher en abril/08 y en $T$. stellatus en enero/08, abril/08 y en junio/08 se debió al consumo de un solo tipo de presa (cladóceros en $C$. pulcher y artrópodos terrestres en T. stellatus). Esto indica que una reducción en la amplitud de dieta no necesariamente estará asociada a una disminución del consumo de presas sino a una variación en las proporciones de consumo.

Los resultados mostraron que en SA los invertívoros dependen de la misma comunidad de presas (insectos acuáticos) ya que el ambiente mantiene su condición lótica mientras que en los invertívoros de SB ocurrió un reemplazo importante de presas posiblemente como consecuencia de las condiciones ambientales adversas para un grupo (insectos acuáticos) pero favorables para otro (zooplancton).

La sobreposición de dieta obtenida puede sub o sobreestimar la real debido a que no considera la posible asimetría en el uso de los recursos. Por ejemplo, el valor obtenido entre $K$. deuteronoides y $P$. reticulata en marzo/08 fue IM: 0.9114. Sin embargo, $K$. deuteronoides compartió con P. reticulata sólo uno de los siete tipos de presas consumidas (14\% de ellas), mientras que $P$. reticulata compartió el único tipo de presa que consumió (100\%), lo cual no quedaría reflejado en el estimado. Al respecto, una opción podría ser incorporar al cálculo de sobreposición, una variable, como el porcentaje de presas comunes consumidas, que permitiría obtener valores corregidos de sobreposición y un valor por especie. A continuación se detalla el procedimiento:

$$
\mathrm{IM}_{\mathrm{spA-spB}}=0.9114
$$

$\mathrm{IM}_{\mathrm{spA}}=0.9114 \times 14 \%=0.1276$

(14\% representa el porcentaje de tipos de presas que la especie A comparte con la especie B).

$$
\mathrm{IM}_{\mathrm{spB}}=0.9114 \times 100 \%=0.9114
$$

(100\% representa el porcentaje de tipos de presas que la especie B comparte con la especie A).

Aunque en ambas secciones se obtuvieron valores altos de sobreposición trófica, la tendencia fue opuesta en casi todo el registro. Otra diferencia fue que en SB pocas estimaciones 
resultaron mayores del 50\% (28\% del total), lo que indica una tendencia a la diferenciación de dieta. Por el contrario, en SA, el $80 \%$ de las estimaciones resultaron mayores al $50 \%$ de sobreposición, lo que indicaría la existencia de dietas más amplias. Esto muestra que hay un gradiente de sobreposición como el reportado para otros ambientes (DeSilva \& Kortmulder 1977, DeSilva et al. 1980, Atencio-García et al. 2005, Alford \& Beckett 2007). Pouilly et al. (2006) encontraron, en la cuenca alta del río Beni (Bolivia), que en aguas bajas los peces consumieron los recursos autóctonos del ambiente (invertebrados), con un bajo consumo de recursos alóctonos (invertebrados terrestres, frutos y semillas). Los autores consideraron que en aguas bajas dominó la especialización hacia el consumo de invertebrados acuáticos. Esto corresponde parcialmente con lo reportado por Winemiller (1990) en la cuenca del río Orinoco, quien reportó el consumo de recursos autóctonos en aguas bajas pero representados principalmente por detritus y vegetación acuática, mientras que en aguas altas los recursos alóctonos (invertebrados terrestres, frutos y semillas) resultaron ser una fuente alimenticia importante.

La sobreposición de dieta obtenida en SB corresponde de manera parcial con reportes de menor sobreposición trófica en aguas bajas, considerada como la de menor disponibilidad de alimento (Zaret \& Rand 1971). Sin embargo, la mayor sobreposición obtenida en SA difiere de lo anterior (Goulding 1980, LoweMcConnell 1987, Ortaz 2001). Esto indica que la interacción dentro de la comunidad variará no sólo por el efecto de la descarga sino también de las características particulares de cada sección que pueden amortiguar en grados variables el impacto de los cambios ambientales. Es de esperar que las interacciones intragremio sean intensas, sin embargo, como se encontró en SB, puede ocurrir diferenciación trófica que reduciría tal intensidad. Sin embargo, en general, la competencia intragremio por el alimento puede ser intensa por ocupar las especies nichos tróficos parecidos, lo que se considera como una poderosa fuerza selectiva que juega un papel fundamental como reguladora de las poblaciones y por medio de la cual se puede promover la evolución inter e intrapoblacional (Ward et al. 2006).

\section{AGRADECIMIENTOS}

Al CDCH-UCV por el financiamiento otorgado a través de un proyecto individual (Proyecto No 03-6857-2007). A Daniel Veroes por su ayuda en los trabajos de campo. A Maria E. Rocha por la revisión de la versión en inglés del resumen.

\section{RESUMEN}

La ictiofauna invertívora es un componente importante en los ríos neotropicales y representa un enlace entre los invertebrados acuáticos y los piscívoros. En el presente trabajo se evaluó la amplitud y sobreposición interespecífica de dieta de nueve especies de peces invertívoros durante tres fases hidrológicas consecutivas: descenso (diciembre/07, enero/08, febrero/08, marzo/08), aguas bajas (abril/08) y ascenso de aguas (junio/08) en dos secciones de un río venezolano ubicadas a distinta altitud (subcuenca alta, SA y subcuenca baja, SB). Los peces se recolectaron con una red de cerco $(0.5 \mathrm{~cm}$ entrenudos) entre las 8:00 y 11:00 horas. La dieta de cada especie se evaluó usando un índice de importancia relativa (IIR) que incluyó el número, peso y frecuencia de ocurrencia de los items consumidos. Para estimar la amplitud y sobreposición interespecífica de dieta se emplearon los índices de Levín ( $\mathrm{B}_{\mathrm{i}}$ ) y Morisita (IM), respectivamente. Todas las estimaciones se realizaron con la frecuencia numérica de las presas consumidas. Se capturaron nueve especies, ocho Characiformes, tres en SA (Knodus deuteronoides, Creagrutus bolivari y C. melasma) y cinco en SB (Thoracocharax stellatus, Moenkhausia lepidura, Cheirodon pulcher, Ctenobrycon spilurus y Aphyocharax alburnus) y un Cyprinodontiformes (Poecilia reticulata), capturada en SA. En SA el principal recurso consumido fueron insectos acuáticos mientras que el material vegetal y los artrópodos terrestres fueron recursos secundarios. En SB, las especies consumieron estos items además de zooplancton (Copepoda, Cladocera y estadios larvales de crustáceos Decapoda), pero hubo una sustitución temporal con un predominio de zooplancton en descenso y aguas bajas. En general, la amplitud de la dieta disminuyó en descenso de aguas en ambas secciones e incrementó en ascenso de aguas. Sin embargo, la amplitud promedio fue mayor en SA. La sobreposición interespecífica de dieta fue alta en SA mientras que en SB fueron más frecuentes los valores bajos y en casi todo el registro su variación temporal fue opuesta en ambas secciones. 
Palabras clave: ecología trófica, Characiformes, peces invertívoros, río neotropical, Venezuela.

\section{REFERENCIAS}

Allan, J.D. 1995. Stream ecology: structure and function of running waters. Chapman \& Hall, Londres, Reino Unido.

Alford, J.B. \& D.C. Beckett. 2007. Selective predation by four darter (Percidae) species on larval chironomids (Diptera) from a Mississippi stream. Environ. Biol. Fish. 78: 353-364.

Angermeier, P.L. \& J.R. Karr. 1983. Fish communities along environmental gradients in a system on tropical streams. Environ. Biol. Fish. 9: 117-135.

Atencio-García, V.J., E. Kerguelen-Durango, E.C. Dorado, R.R. Carcomo, A. Vallejo \& M. Valderrama. 2005. Régimen alimentario de siete especies ícticas en el embalse de la hidroeléctrica Urrá (Córdova, Colombia). Publ. Fac. Medicina, Veterinaria y Zootecnia 10: 614-622.

Barbee, N.C. 2005. Grazing insects reduce algal biomass in a neotropical stream. Hydrobiologia 532: 153-165.

Calow, P. \& G.E. Petts. 1992. The river handbook. Vol. II. Blackwell, Londres, Reino Unido.

Chesson, J. 1978. Measuring preference in selective predation. Ecology 59: 211-215.

DeSilva, S.S. \& K. Kortmulder. 1977. Some aspects of the biology of three species of Puntius (Barbus) (Pisces: Cyprinidae), endemic to Sri Lanka. Neth. J. Zool. 27: 182-194.

DeSilva, S.S., K. Kortmulder \& M.J. Wijeyrathe. 1980. A comparative study of the food and feeding habits of Puntius bimaculatus and P. titteya (Pisces: Cyprinidae). Neth. J. Zool. 27: 253-263.

Fernández, H.R. \& E. Domínguez. 2001. Guía para la determinación de los artrópodos bentónicos sudamericanos. Universidad Nacional de Tucumán, Tucumán, Argentina.

Flecker, A.S. 1992. Fish predation and the evolution of invertebrate drift periodicity: evidence from neotropical streams. Ecology 73: 438-448.

Géry, J. 1977. Characoids of the world. TFH, Neptune City, EEUU.
Goulding, M. 1980. The fishes and the forest. Exploration Amazonian Natural History. Universidad de California, Los Angeles, EEUU.

Horn, H. 1966. Measurement of overlap in comparative ecological studies. Am. Nat. 100: 420-424.

Hyslop, E.J. 1980. Stomach contents analysis - a review of methods and their application. J. Fish. Biol. 17: 411-429.

Lowe-McConnell, R.H. 1987. Ecological studies in tropical fish communities. Cambridge, Londres, Reino Unido.

Machado-Allison, A. 2005. Los peces de los Llanos de Venezuela: un ensayo sobre su historia natural. Universidad Central de Venezuela, Caracas, Venezuela.

Machado-Allison, A. \& H. Moreno. 1993. Estudios sobre la comunidad de peces del río Orituco, Estado Guárico, Venezuela. Parte I. Inventario, abundancia relativa y diversidad. Acta. Biol. Venezuélica 14: 77-94.

Martín, R. 2007. Estrategia alimentaria de algunas especies de carácidos (Pisces: Characidae) en una sección de la cuenca baja del río Orituco (Estado Guárico, Venezuela). Tesis de Licenciatura, Universidad Central de Venezuela, Caracas, Venezuela.

Mérigoux, S. \& D. Porton. 1998. Body shape, diet and ontogenetic diet shifts in young fish of the Sinnamary river, French Guiana, South America. J. Fish. Biol. 52: 556-569.

Merritt, R.W. \& K.W. Cummins. 1984. An introduction to the aquatic insects of North America. Kendall/Hunt, Duduque, EEUU.

Moyle, P.B. \& F.R. Senanayake. 1984. Resource partitioning among fishes of rainforest stream in Sri Lanka. J. Zool. 202: 195-224.

Ortaz, M. 1992. Hábitos alimentarios de los peces de un río de montaña neotropical. Biotropica 37: 298-301.

Ortaz, M. 2001. Diet seasonality and food overlap among fishes of the upper Orituco stream, northern Venezuela. Rev. Biol. Trop. 49: 191-197.

Ortaz, M. \& M.E. Rocha. 2001. Dieta de Creagrutus melasma (Pisces: Characidae) en un río de montaña de zona boscosa. Acta. Biol. Venezuélica 21: 9-15.

Ortaz, M., P. Borjas von Bach \& R. Candia. 2006. Alimentación del pez insectívoro neotropical Creagrutus bolivari (Pisces: Characidae) según los métodos gráfico y de importancia relativa. Rev. Biol. Trop. 54: 1227-1239. 
Pouilly, M., F. Lino, J.G. Bretenoux \& C. Rosales. 2003. Dietary-morphological relationships in a fish assemblage of the Bolivian Amazonian floodplain. J. Fish. Biol. 62: 1137-1158.

Pouilly, M., S. Barrera \& C. Rosales. 2006. Changes of taxonomic and trophic structure of fish assemblages along an environmental gradient in the Upper Beni watershed (Bolivia). J. Fish. Biol. 68: 137-156.

Roldán, P.G. 1996. Guía para el estudio de los macroinvertebrados acuáticos del Departamento de Antioquia. Fondo Fen Colombia, Conciencias, Universidad de Antioquia, Bogotá, Colombia.

Smith, E.P. 1985. Estimating the reliability of diet overlap measures. Environ. Biol. Fish. 13: 125-138.

Taphorn, D. 1992. The characiform fishes of the Apure river drainage, Venezuela. Biollania 4: 1-537.

Thorp, J.H. \& M.D. Delong. 1994. The riverine productivity model: an heuristic view of carbon sources and organic processing in large river ecosystems. Oikos 70: 305-308

Trujillo-Jiménez, P. \& H. Toledo-Beto. 2007. Alimentación de los peces dulceacuícolas tropicales Heterandria bimaculata y Poecilia sphenops (Cyprinodontiformes: Poeciliidae). Rev. Biol. Trop. 55: 603-615.

Vannote, R.L., G.W. Minshall, K.W. Cummins, J.R. Sedell \& C.E. Cushing. 1980. The river continuum concept. Can. J. Fish. Aquat. Sci. 37: 130-137.
Ward, A.J., M.M. Webster \& P.J. Hart. 2006. Intraspecific food competition in fishes. Fish Fish. 7: 231-261.

Welcomme, R.L. 1985. River fisheries. FAO, Roma, Italia.

Wetzel, R. 2001. Limnology: lake and river ecosystems. Elsevier, San Diego, EEUU.

Wetzel, R.G. \& G.E. Likens. 2000. Limnological analyses. Springer, Nueva York, EEUU.

Winemiller, K.O. 1990. Spatial and temporal variation in tropical fish trophic networks. Ecol. Monographs 60 331-367.

Winemiller, K.O. 2005. Floodplain river food webs: generalization and implications for fisheries management, p. 285-312. In R.L. Welcomme \& T. Petr (eds.). Proceeding of the second international symposium on the management of large rivers for fisheries. Vol. 2. Mekong River Commission, Phnom Penh, Cambodia.

Wootton, R.J. 1990. Ecology of teleost fishes. Chapman \& Hall, Londres, Reino Unido.

Zaret, T.M. \& E.P. Smith. 1984. On measuring niches and not measuring them, p. 127-137. In T.M. Zaret (ed.). Evolutionary ecology of neotropical freshwater fishes. Dr. Junk, Boston, EEUU.

Zaret, T.M. \& A.J. Rand. 1971. Competition in tropical stream fish communities. Ecology 59: 507-515. 